\title{
Vitamin D and platelet Rich Plasma (PRP) in the treatment of vitiligo
}

Talal Ahmed Abd El Raheem ${ }^{(1)}$, Basma Hamada Mohammed ${ }^{(2)}$, Noha Tolba Mostafa El- Sayed $^{(3)}$.

(1) Professor of Dermatology,STDs and Andrology ,Faculty of Medicine, Fayoum University>

(2) Lecturer of Dermatology ,Faculty of Medicine, Fayoum University

(3) M.B.B.CH

\section{ABSTRACT}

Vitamin D and Platelet Rich Plasma (PRP) in the treatment of Vitiligo

Background: Vitiligo is an autoimmune disorder which is caused by the destruction of melanocytes in the skin. Vitamin D is a fat-soluble vitamin that controls cell proliferation and differentiation and exerts immunoregulatory activities. PlateletRich Plasma (PRP) may serve as a source of different growth factors to induce pigmentation in vitiligo.

Aim of the work: to explore the effect of intralesional platelet rich plasma and vitamin $\mathrm{D}$ injection in vitilgo patients.

Patients and methods: The study included two groups of stable non segmental vitiligo patients. Each group included 20 patients.

Group A: Each patient was injected PRP intralesionaliy in vitiligenous patch and PRP together with vitamin D intralesionally in another vitiligenous patch.

Group B : Each patient was injected vit $\mathrm{D}$ intralesionally in vitiligenous patch and PRP together with vitamin D intralesionally in another vitiligenous patch .

\section{Results:}

Group A revealed highly statistically significant difference between baseline and after 3 months follow up regarding improvement of pigmentation in both PRP +vit. D patch $(\mathrm{p}<0.001)$ and in PRP group $(\mathrm{p}<0.001)$.

Group B revealed highly statistically significant difference between baseline and after 3 months follow up regarding degree of improvement of pigmentation in PRP + vit D group ( $p<0.001)$. It revealed statistically significant difference between baseline and after 3 months follow up regarding degree of improvement of pigmentation in vit $\mathrm{D}$ group $(\mathrm{p}<0.05)$.

Conclusion: In conclusion, PRP injection either alone or with vit. D has good satisfactory results in treatment of vitilig. Vit.D injection alone showed less satisfactory results than combined treatment regarding degree of improvement.

KEYWORDS: Vitiligo,Vitamin D and Platelet Rich Plasma. 


\section{INTRODUCTION :}

Vitiligo is an autoimmune disorder which is caused by the destruction of melanocytes in the skin. ${ }^{[1]}$ The disease may affect both genders and all skin types and may also be associated with systemic autoimmune diseases such as lupus erythematosus, scleroderma, autoimmune thyroiditis and alopecia areata. ${ }^{[2]}$

Vitiligo presents as well circumscribed, depigmented macules and patches with convex borders, surrounded by normal skin which is slowly progressive. It can be subdivided into non segmental vitilgo,segmental vitilgo and unclassified vitiligo. ${ }^{[3]}$

No single therapy for vitiligo produces predictably good results in all patients and the response to therapy is highly variable.$^{[4]}$ Treatment options include topical treatment which include topical corticosteroids, vitamin D analogues and topical calcineurin inhibitors while systemic treatment options include corticosteroids and immunosuppressive, phototherapy including narrowband ultraviolet $\mathrm{B}$, psoralen with ultraviolet $\mathrm{A}$

\section{PATIENTS AND METHODS:}

. Forty adult patients with localized non segmental vitiligo were enrolled in this study. They were recruited from the Outpatient Clinic of Dermatology and Venereology Department, Fayoum University Hospital.

\section{Inclusion criteria:}

1-Age above 12 years.

2-Localized stable non segmental vitiligo.

\section{Exclusion criteria:}

1-Patients below 12 years.

2-Pregnant and lactating females. along with surgical treatment, in addition to camouflage cosmetics and sunscreens [5]

Vitamin $\mathrm{D}$ is a fat-soluble vitamin obtained by humans through diet. It has been used to treat psoriasis, vitiligo and other skin diseases for many years ${ }^{[6]}$ It controls cell proliferation and differentiation and exerts immunoregulatory activities . [1]

Platelet-rich plasma (PRP) is an autologous preparation of platelets in concentrated plasma. Various growth factors are secreted from $\alpha$-granules of concentrated platelets activated by aggregation inducers . ${ }^{[7]}$

Various studies have proved the clinical applications and results of PRP in the fields of dermatology. ${ }^{[8]}$ PRP has been used to treat acne, scarring, and alopecia. It is also effective for skin rejuvenation and tightening around the eyes . [9]

The aim of this study is to compare the effect of intralesional PRP and vitamin D injection in vitilgo patients.

\section{3- Patients with segmental vitilgo.}

4-Patients with koebner phenomen.

5-Patients with chronic systemic diseases (anemia, Diabetes mellitus, immunosuppressed patients, chronic infection and patients with other autoimmune disease as thyroiditis).

\section{-subjects:}

- The patients were randomly categorized into 2 groups.

- Each group included 20 patients.

- Group A: PRP was injected intradermally using insulin syringe in the 
lesion, $0.1 \mathrm{cc}$ of PRP was injected per point with a space of $0.5 \mathrm{~cm}$ between different points of injections in one patch of the body and PRP alternatively with vitamin $\mathrm{D}$ intralesionally in another vitiligenous patch $(0.1 \mathrm{cc}$ of vit. $\mathrm{D}$ was injected per point with a space of $0.5 \mathrm{~cm}$ between different points of injections).

- Group B: By the almost used protocol, each patient was injected $0.1 \mathrm{cc}$ of vit.D per point with a space of $0.5 \mathrm{~cm}$ between different points of injections in one patch of the body and PRP alternatively with vitamin D intralesionally in another vitiligenous patch.

-Three sessions were done one month apart. A digital photography taken before initial and after each session by same camera

-Follow up after three months to detect results any recurrence and any associated side effects

\section{PRP preparation method:}

To create PRP, 4 to $10 \mathrm{cc}$ of venous blood had been collected from the anticubital vein using $21 \mathrm{G}$ sterile $10 \mathrm{ml}$ syringe under complete aseptic conditions. The whole blood sample was collected in plastic tubes containing sodium citrate (10:1) as an anticoagulant (to bind calcium and prevent the initiation of the clotting cascade by preventing the conversion of prothrombin to thrombin).

Then the citrated whole blood was subjected to two centrifugation steps using $800 \mathrm{D}$ centrifuge. The initial centrifugation was done ("soft" spin) at $500 \mathrm{rpm}$ for $10 \mathrm{~min}$ to separate the plasma and platelets from the red and white cells.The resulting plasma supernatant, which contained the suspended platelets (and may contain a portion of the white cell "buffy coat") was harvested to a second centrifugation step ("hard" spin) in plain plastic tubes at $2500 \mathrm{rpm}$ for $15 \mathrm{~min}$,

\section{PRP group:}

Patients received 3 sessions of autologous intradermal PRP injection with a month interval. Topical anesthetic cream (Pridocaine ${ }^{\circledR}$ ) was applied under occlusion 30 minutes before the session and then the skin was sterilized by alcohol. The area was injected with $1 \mathrm{ml}$ insulin syringe $0.5 \mathrm{~cm}$ spacing between injection sites, with maximum of $1 \mathrm{~mL} / \mathrm{session}$. The patients were followed up for

3 months

after the last session

\section{Vitamin D injection:}

Patients received 3 sessions of intradermal Cholecalciferol (Vit. D3) $\left(\right.$ Devarol-S $^{\mathrm{R}}$ ) injection with one month interval. Topical anesthetic cream (Pridocaine ${ }^{\circledR}$ ) was applied under occlusion 30 minutes before the session and then the skin was sterilized by alcohol. Injection was intralesional $0.5 \mathrm{~cm}$ spacing between injection sites, with maximum of $1 \mathrm{~mL} / \mathrm{session}$ using $1 \mathrm{ml}$ insulin syringe. The patients were followed up once after 3 months after the last session.

\section{Evaluation of the treatment:}

Photographs were obtained at baseline, before each treatment session, and 3 months after the final treatment. Objective clinical assessments of repigmentation were performed by 2 blinded dermatologists.

-Scoring system of degree of repigmentation:

- $\mathrm{Go}=$ no improvement.

- $\mathrm{G} 1=<25 \%$ repigmentation. 
- $\mathrm{G} 2=25 \%-50 \%$ repigmentation.

- $\mathrm{G} 3=50 \%-75 \%$ repigmentation.

- $\mathrm{G} 4=>75 \%$ repigmentation.

-Pattern of repigmentation:

- Perifollicular.

- Marginal.

- Mixed.

\section{Patient satisfaction:}

Patients were asked for his satisfaction about results of sessions and their compliance:

- Not satisfied

- Low satisfaction

- High satisfaction

\section{Safety assessment:}

The patients were informed to report any complications as; erythema, pain, ulceration, burning sensation, ecchymosis, infection, postinflammatory hyperpigmentation, or any allergic manifestations.

\section{Statistical methods:}

The collected data were coded, fed to computer, organized and statistically analyzed. Statistical analysis was done using computer programs: Microsoft excel version 10 and Statistical Package for Social Science (SPSS) for windows version $25.00^{[10]}$

\section{Descriptive statistics:}

1-Qualitative (categorical) data were presented by frequency and percentage.

Qualitative data were subjected to Categorical variables are given as number and percentages. A chi-square test.

2- Quantitative data were presented by mean \pm SD.

Group A revealed highly statistically significant difference between Baseline and after 3 months follow up regarding improvement of pigmentation in PRP +vit. D patch $(\mathrm{p}<0.001)$. 45\% (9) patients showed no improvement, $15 \%$ (3) of patients showed improvement less than 25\%, 20\%(4) patients showed improvement $25-50 \%, 15 \%$ (3) patients showed improvement $50-75 \%$ and $5 \%$ (1) patient showed improvement $>75 \%$ 


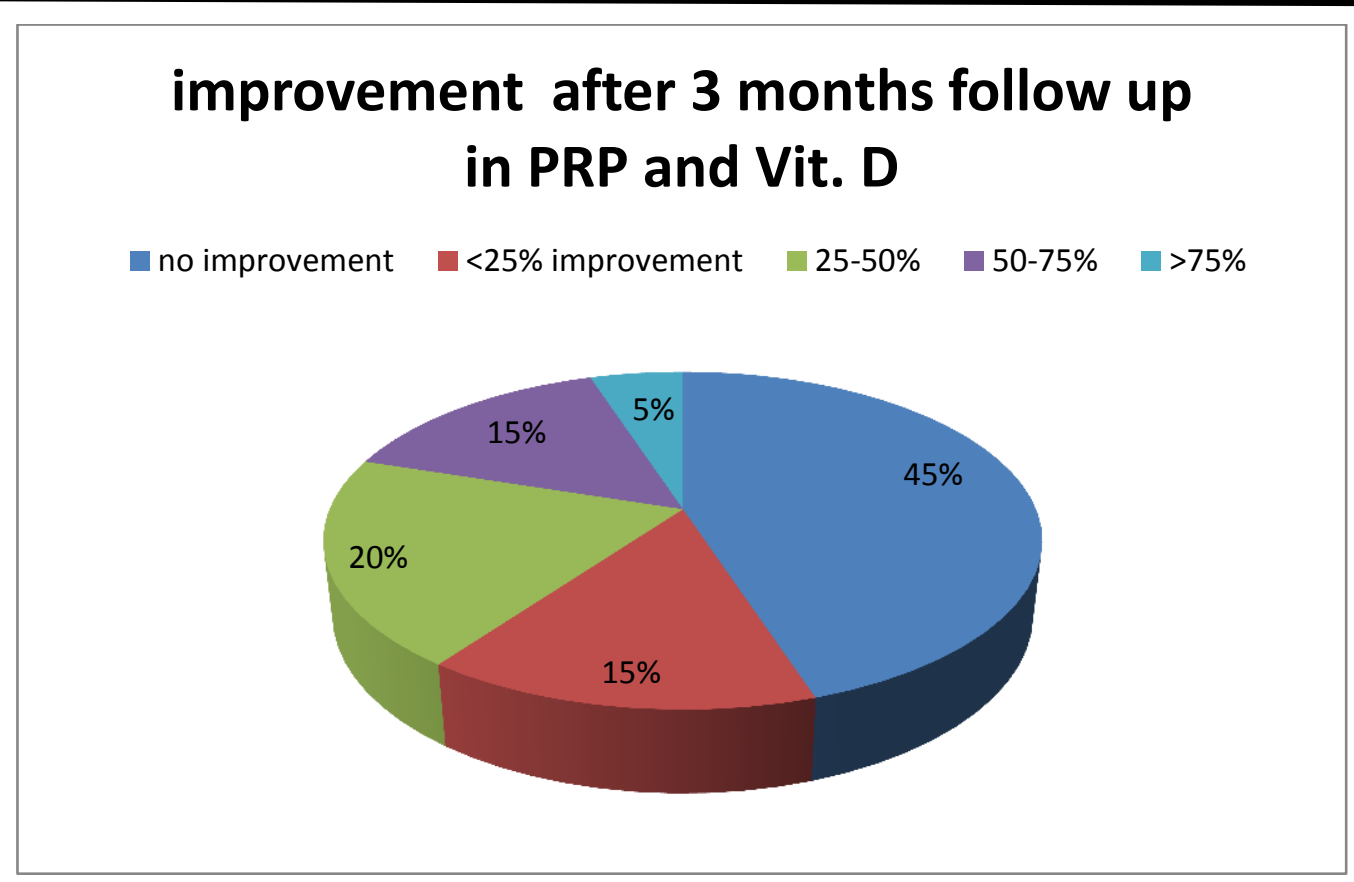

Improvement after 3 months follow up regarding degree of improvement of pigmentation in PRP + vit. D patch ,

-No recurrence of depigmentation in patients after 3 months follow up.

Group A revealed highly statistically significant difference between Baseline and after 3 months follow up regarding degree of improvement of pigmentation in PRP group ( $\mathrm{p}<0.001) .40 \%$ (8) patients showed no improvement , $15 \%$ (3) patients showed improvement less than $25 \%, 10 \%$ (2) patients showed improvement $25-50 \%$, 30\% (6) patients showed improvement $50-75 \%$ and $5 \%$ (1) patient showed improvement $>75 \%$ 


\section{improvement after $\mathbf{3}$ months follow up in PRP patch}

ano improvement $\quad-<25 \% \quad \square 25-50 \% \quad \square 50-75 \% \quad \square>75 \%$

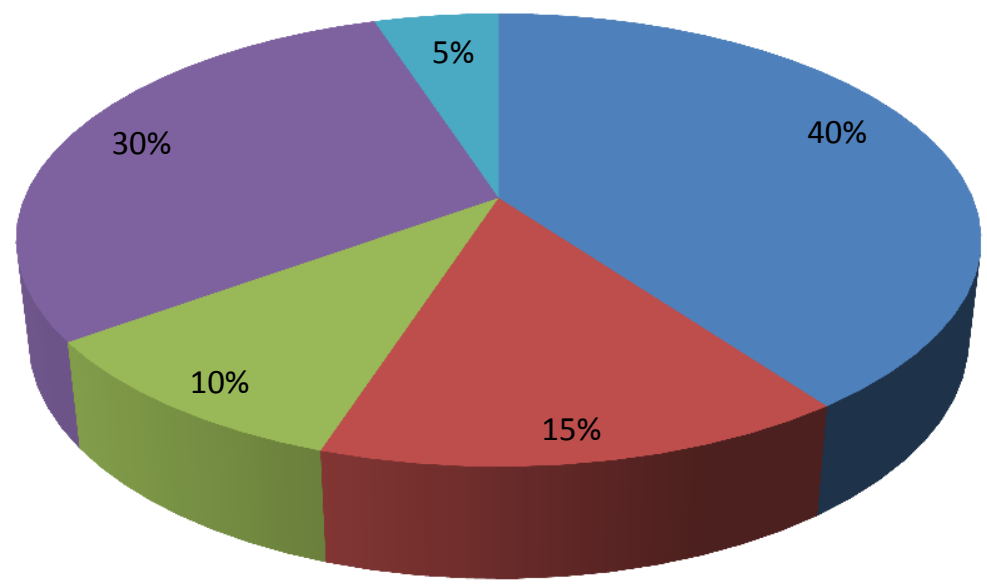

Improvement after 3 months follow up regarding improvement of pigmentation in PRP patch.

-No recurrence of depigmentation in patients after 3 months follow up.

Group B revealed highly statistically significant difference between baseline and after 3 months follow up regarding degree of improvement of pigmentation in PRP + Vit D group ( $\mathrm{p}<0.001) .40 \%$ (8) patients showed no improvement, $10 \%$ (2) patients showed improvement less than $25 \%, 35 \%$ (7) patients showed improvement $25-50 \%, 10 \%$ (2) patients showed improvement 50-75\% and 5\% (1) patient showed improvement $>75 \%$ 


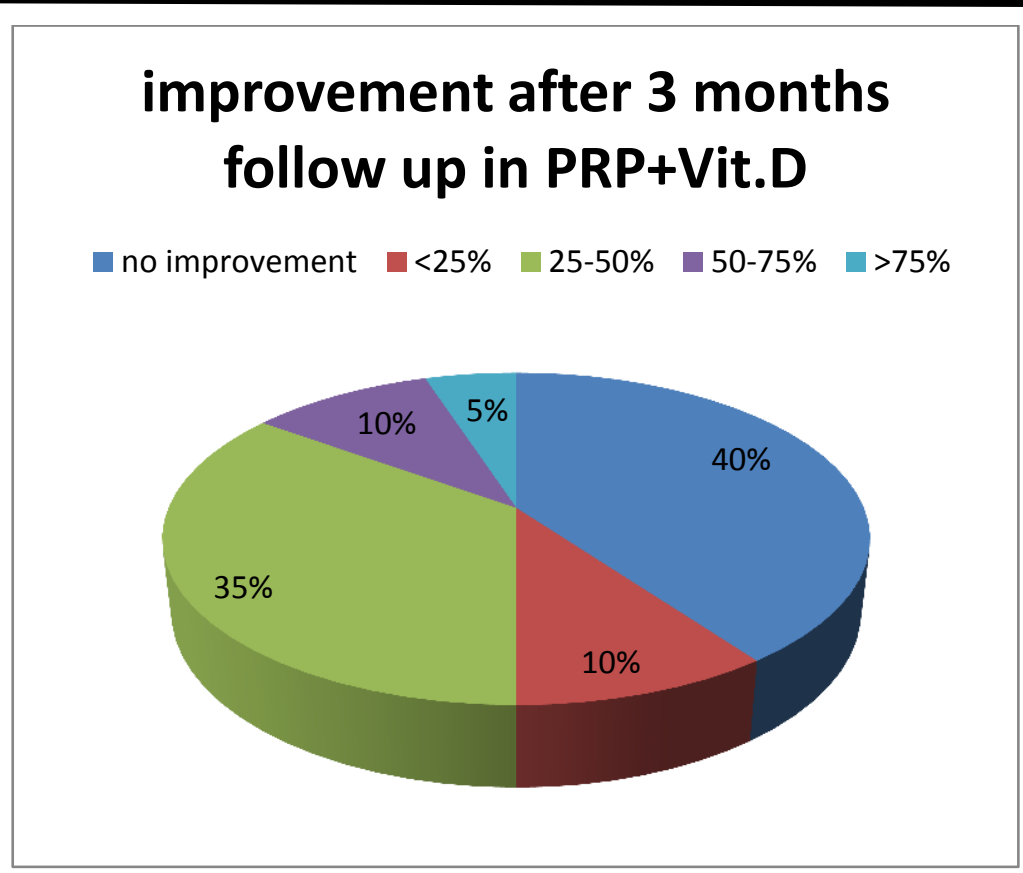

Improvement after 3 months follow up regarding improvement of pigmentation in PRP + Vit D patch.

-No recurrence of depigmentation after 3 months follow up.

Group B revealed statistically significant difference between baseline and after 3 months follow up regarding degree of improvement of pigmentation in Vit $\mathrm{D}$ group ( $\mathrm{p}<0.05)$. 60\% (12) patients showed no improvement, $0 \%(0)$ patients showed improvement less than

\section{DISCUSSION}

Vitiligo is an autoimmune disorder which is caused by the destruction of melanocytes in the skin.$^{[1]}$

No single therapy for vitiligo produces predictably good results in all patients and the response to therapy is highly variable ${ }^{4}$ Treatment options include topical treatment which include topical corticosteroids, vitamin D analogues and topical calcineurin inhibitors while systemic treatment options include
$25 \%, \quad 15 \%(3) \quad$ patients showed improvement $25-50 \%$, 20\% (4) patients showed improvement $50-75 \%$ and $5 \%$ (1) patient showed improvement $>75 \%$

Improvement after 3 months follow up regarding degree of improvement of pigmentation in Vit D patch

No recurrence of depigmentation after 3 months follow up.

corticosteroids and immunosuppressive, photothrapy including narrowband ultraviolet B, psoralen with ultraviolet A along with surgical treatment, in addition to camouflage cosmetics and sunscreens. ${ }^{[5]}$

Vitamin D is a fat-soluble vitamin obtained by humans through diet. It has been used to treat psoriasis, vitiligo and other skin diseases for many years ${ }^{6}$ It controls cell proliferation and 
differentiation and exerts

immunoregulatory activities.$^{[1]}$

Platelet-rich plasma (PRP) is an autologous blood-derived product enriched in platelets, growth factors, chemokines, and cytokines. It is a reservoir of essential growth factors. Various studies have proved the clinical applications and results of PRP in the fields of dermatology. ${ }^{8}$ PRP has been used to treat acne, scarring, and alopecia. It is also effective for skin rejuvenation and tightening around the eyes . ${ }^{[9]}$

The aim of our study was to explore the effect of intralesional platelet rich plasma and vitamin D injection in vitilgo patients.

Our study included two groups of stable non segmental vitiligo patients. Each group included 20 patients.

- Group A: included 17 female and 3 males their age ranged from 13-42 years (mean \pm SD 23.40 \pm 9.25).

Using insulin syringe, PRP was injected intradermally , $0.1 \mathrm{cc}$ per point $0.5 \mathrm{~cm}$ apart in one patch of the body and PRP alternatively with $0.1 \mathrm{cc}$ Vit. D $0.5 \mathrm{~cm}$ apart in another vitiligenous patch.

- Group B: included 9 female and 11 males their age ranged from 12-45years (mean \pm SD 24.45 \pm 10.72).

Using insulin syringe, vit.D was injected intradermally, $0.1 \mathrm{cc}$ per point $0.5 \mathrm{~cm}$ apart in one patch of the body and $0.1 \mathrm{cc}$ PRP alternatively with vit. D 0.5 $\mathrm{cm}$ apart in another vitiligenous patch.

-Three sessions were done one month apart. A digital photography was taken before initial and after each session for evaluation clinically together with measurement of degree of improvement and pattern of repigmentation.Follow up after three months to detect any possible recurrence .

For our knowledge it is the first study to be done in Egypt to treat vitiligo with combined intralesional injection of PRP and vitamin $\mathrm{D}$.

\section{Group A}

In our study, at 3 months follow up group A showed 55\% improvement in PRP and vit. D and 60\% improvement in PRP patches.

It revealed highly statistically significant difference between baseline and after 3 months follow up regarding improvement of pigmentation in both PRP + vit. D patch and PRP only patch $(\mathrm{p} \leq 0.001)$.

In PRP and vit.D patch, $45 \%$ of patients showed no improvement , $15 \%$ showed less than $25 \%$ improvement, $20 \%$ of patients showed $25-50 \%$ improvement, $15 \%$ showed $50 \%-75 \%$ improvement and 5\% showed improvement more than $75 \%$.

In PRP patch, $40 \%$ showed no improvement, $15 \%$ showed less than $25 \%$ improvement, $10 \%$ showed $25 \%$ $50 \%$ improvement , $30 \%$ of patients showed 50\%-75\% improvement and only $5 \%$ showed improvement more than $75 \%$ and this is in consistent with Ibrahim et al., $2016{ }^{[11]}$ who studied 60 stable vitiligo patients with overall symmetrical lesions and found that in the PRP side, 33 patients (55\%) had 75\%-100\% improvement, 12 patients $(20 \%)$ had $50 \%-75 \%$ improvement, 9 (15\%) patients had $25 \%-50 \%$, and $6(10 \%)$ patients had less than $25 \%$ improvement.

Also, Abdelghani et al., ${ }^{[12]}$ studied eighty adult patients with localized nonsegmental vitiligo .and found that PRP group together with fractional $\mathrm{Co} 2$ laser achieved the best results regarding 
repigmentation and patient's satisfaction which was similar to our patients satisifaction where we found $20 \%$ of patients not satisified, $40 \%$ with low satisifaction and $40 \%$ with high satisifaction in PRP and vit.D patches, while in PRP patches $15 \%$ not satisfied , 30\% of them were low satisfied and $55 \%$ were highly satisfied with the results.

In contrast with $\mathbf{L i m}$ et al., ${ }^{[13]}$ in who treated 20 patients with vitiligo by intradermal injection of PRP weekly for 10 weeks and they suggested that PRP was not effective in the treatment of vitiligo.

\section{Group B}

Platelet rich plasma and vit.D patches showed $60 \%$ improvement while vit.D patches revealed only $40 \%$ improvement after 3 months follow up.

It revealed highly statistically significant difference between Baseline and after 3 months follow up regarding degree of improvement of pigmentation in Prp + vit D group $(\mathrm{p} \leq 0.001)$ and It revealed statistically significant difference between Baseline and after 3 months follow up regarding degree of improvement of pigmentation in vit $\mathrm{D}$ group $(\mathrm{p} \leq 0.05)$.

Forty \% Of patients of PRP and Vit. D patch showed no improvement, $10 \%$ revealed less than $25 \%$ improvement , 35 $\%$ showed improvement between 25 $50 \%, 10 \%$ showed $50 \%-75 \%$ improvement and only 5\% showed more than $75 \%$ improvement.

In vit.D patches, $60 \%$ of them were not improved at all, $15 \%$ of showed $25-50 \%$ improvement, $20 \%$ showed $50-75 \%$ improvement and only $5 \%$ improved more than $75 \%$.
It revealed no statistically significant difference between PRP + vit D and vit D regarding degree of improvement of pigmentation after 3 months follow up ( $\mathrm{p}$ $>0.05$ ).

In group B , 10 patients (83\%) revealed perifollicular repigmentation, 0 patients $(0 \%)$ revealed marginal repigmentation and 2 patients $(17 \%)$ revealed mixed repigmentation.

However, in vit.D patches 5 patients $(62.5 \%)$ revealed perifollicular repigmentation, 1 patients $(12.5 \%)$ revealed marginal repigmentation and 2 patients $(25 \%)$ revealed mixed repigmentation.

No recurrence of depigmentation. No Koebner phenomenon occurred, no other complications during follow up period.

Regarding patients satisfaction in PRP and vit.D patches $25 \%$ of patients were not satisfied, $40 \%$ with low satisifaction and $35 \%$ of them with high satisifaction and in vit.D patches $40 \%$ of patients were not satisfied, $25 \%$ with low satisfaction and $35 \%$ of them with high satisfaction.

In this study, we prepared PRP by double-spin centrifugation protocol.

This method of preparation was chosen on the basis that double-centrifugation protocol using the correct g-forces and spin times results in higher platelet concentrations than the single centrifugation protocol.

In this study, We partially applied the protocol done by Abdelghani et al., ${ }^{[12]}$ and Ibrahim et al., ${ }^{[11]}$ they recommended double centrifugation.

In this study, We inject patients once per month in contrast to Abdelghani et al., ${ }^{[12]}$ and Ibrahim et al., ${ }^{[11]}$ who injected every weeks. 
For our knowledge we are the first to inject vit. D intralesional in vitiligo patients.A number of studies have been reported the treatment of vitiligo with oral and topical vitamin D analogues alone or in combination with ultraviolet light or corticosteroids to enhance repigmentation. ${ }^{[1]}$

In conclusion, PRP injection either alone or with vit. D has good satisfactory RECOMMENDATIONS:

1-Platelet rich plasma in vitiligo treatment requires further assessement and more studies.

2-Combination of other lines of treatment with PRP may be required to get higher degree of improvement.

3-Injection at short interval e.g every two weeks.

\section{REFERENCES:}

[1] AlGhamdi K, Kumar A and Moussa $\mathrm{N}$. The role of vitamin $\mathrm{D}$ in melanogenesis with an emphasis on vitiligo. Indian $J$ Dermatol Venereol Leprol 79,750-758 (2013).

[2] Lee S, Zheng Z, Kang J, Kim D, Oh S and Bin $S$.Therapeutic efficacy of autologous platelet-rich plasma and polydeoxyribonucleotide on female pattern hair loss. Wound Repair Regen 23,30-36 (2015) .

[3] Alikhan A, Felsten LM, Daly M and Petronic-Rosic V: Vitiligo.a comprehensive overview Part I.Introduction, epidemiology, quality of life, diagnosis, differential diagnosis, associations, histopathology, etiology, and work-up.J Am Acad Dermatol 65,473-491 (2011).

[4] Ohguchi R, Kato H, Furuhashi T, Nakamura M, Nishida E, Watanabe S, Shintani Y and Morita A Risk factors results in treatment of vitiligo. It showed repigmentation in vitiligenous patches and improvement of already present pigment (50\% improvement in patients). But we don't advise to use vit.D injection alone as results were less satisfactory than combined treatment regarding degree of improvement.

In conclusion, PRP injection either alone or with vit. D has good satisfactory results in treatment of vitiligo. It show repigmentation in vitiligenous patches and improvement of already present pigment (50\% improvement in patients). But vit.D injection alone results were less satisfactory than combined treatment regarding degree of improvement.

and treatment responses in patients with vitiligo in Japan-A retrospective largescale study. Kaohsiung J Med Sci. 31,260-264 (2015).

[5] Gawkrodger DJ, Ormerod AD, Shaw L, Mauri-Sole I, Whitton ME, Watts MJ, Anstey AV, Ingham $\mathrm{J}$ and Young $\mathrm{K}$ Guideline for the diagnosis and management of vitiligo. $\mathrm{Br} J$ Dermatol. Br J Dermatol 159,1051-1076(2008).

[6] Ustun I, Seraslan G, Gokce C, Motor $\mathrm{S}$, Can Y, Ugur Inan $\mathrm{M}$ and Yilmaz $\mathrm{N}$ Investigation of vitamin $\mathrm{D}$ levels in patients with vitiligo vulgaris. Acta Dermatovenerol Croat 22, 110-113 (2014).

[7] Kaux JF, Le Goff C, Seidel L, Péters P, Gothot A, Albert A and Crielaard JM .Comparative study of five techniques of preparation of platelet-rich plasma]. Pathol Biol (Paris)59,157-160. (2011) . 
[8] Perez AG, Lana JF, Rodrigues AA, Luzo AC, Belangero WD and Santana $\mathrm{MH}$.Relevant aspects of centrifugation step in the preparation of platelet-rich plasma. ISRN Hematol; 76060. (2014) .

[9] Shin J, Lee JS, Hann SK and Oh SH Combination treatment by $10600 \mathrm{~nm}$ ablative fractional carbon dioxide laser and narrowband ultraviolet $\mathrm{B}$ in refractory nonsegmental vitiligo: a prospective, randomized half-body comparative study. $\mathrm{Br} \quad J$ Dermatol.166,658-661 (2012) .

[10] Snedecor, G. W. \& Cochran, W. G. Statistical methods. (Iowa State University Press, 1982).

[11] Ibrahim ZA, El-Ashmawy AA, ElTatawy RA and Sallam FA The effect of platelet-rich plasma on the outcome of short-term narrowband-ultraviolet $B$ phototherapy in the treatment of vitiligo: a pilot study. J Cosmet Dermatol. 15,108116 (2016) .

[12] Abdelghani R, Ahmed NA and Darwish HM. Combined treatment with fractional carbon dioxide laser, autologous platelet-rich plasma, and narrow band ultraviolet $\mathrm{B}$ for vitiligo in different body sites: A prospective, randomized comparative trial. $J$ Cosmet Dermatol. 17,365-372 (2018) .

[13] Lim HK, Sh MK and Lee MH . Clinical application of PRP in vitiligo: a pilot study. Official 1st International Pigment Cell Conference 2011. 